\title{
ON SOME PROPERTIES OF THE NORMAL AND GAMMA DISTRIBUTIONS
}

R. G. LAHA

It is well-known [2] that in a normal population any translationinvariant statistic is stochastically independent of the sample mean. Similarly [3] in a gamma population the sample mean is distributed independently of any scale-invariant statistic.

In the present note we shall prove the following theorems.

TheOREM 1. Let $x_{1}, x_{2}, \cdots, x_{n}$ be $n$ identically and independently distributed normal variables. The necessary and sufficient condition that the sum $x_{1}+x_{2}+\cdots+x_{n}$ is distributed independently of some function $g\left(x_{1}, x_{2}, \cdots, x_{n}\right)$ is that $g\left(x_{1}, x_{2}, \cdots, x_{n}\right)$ and $g\left(x_{1}+\lambda, x_{2}+\lambda\right.$, $\left.\cdots, x_{n}+\lambda\right)$ should be identically distributed.

Proof of Necessity. From the conditions of Theorem 1, it follows that

$$
E\left(\exp \left(i t \sum x+i u g\right)\right)=E\left(\exp \left(i t \sum x\right)\right) \cdot E(\exp (i u g))
$$

where $i=(-1)^{1 / 2}$ as usual and $t$ and $u$ are real.

Without any loss of generality, we may assume $x$ 's to be distributed normally with zero mean and unit variance.

Then (1) gives

$$
\begin{aligned}
E\left\{\exp \left(i u g\left(x_{1}, x_{2}, \cdots x_{n}\right)\right)\right\} & \\
= & \frac{1}{(2 \pi)^{n / 2}} \int_{-\infty}^{\infty} \cdots \int_{-\infty}^{\infty} \exp \left(\operatorname{iug}\left(x_{1}, x_{2}, \cdots x_{n}\right)\right. \\
& \left.-\frac{1}{2} \sum(x-i t)^{2}\right) d x_{1} \cdots d x_{n}=\phi(t, u), \text { say. }
\end{aligned}
$$

Now from (2) it follows that $\phi(t, u)$ does not involve $t$ for all real values of $t$.

But it is evident that $\phi(t, u)$ is an entire function in $t$ and hence it should be free of $t$ for all complex values of $t$ also.

Hence putting $t=-i \lambda$ ( $\lambda$ any real number) we have, after some simplifications,

$$
\phi(-i \lambda, u)=E\left\{\exp \left(i u g\left(x_{1}+\lambda, \cdots, x_{n}+\lambda\right)\right)\right\} .
$$

Thus combining (2) and (3) we have

Received by the editors July 12, 1954 and, in revised form, April 10, 1955. 


$$
\begin{aligned}
E\left\{\exp \left(\operatorname{iug}\left(x_{1}, x_{2}, \cdots, x_{n}\right)\right)\right\} \\
=E\left\{\exp \left(i u g\left(x_{1}+\lambda, x_{2}+\lambda, \cdots, x_{n}+\lambda\right)\right)\right\} .
\end{aligned}
$$

Then using the uniqueness theorem of characteristic functions [1], it follows from (4) that $g\left(x_{1}, x_{2}, \cdots, x_{n}\right)$ and $g\left(x_{1}+\lambda, x_{2}+\lambda, \cdots\right.$, $\left.x_{n}+\lambda\right)$ should be identically distributed.

Proof of Sufficiency. As before let us assume $x$ 's to be distributed normally with zero mean and unit variance.

Then the characteristic function of the joint cumulative distribution of $\sum x$ and $g\left(x_{1}, x_{2}, \cdots, x_{n}\right)$ is given by

$$
\begin{aligned}
E(\exp (i t & \left.\left.\sum x+i u g\right)\right) \\
= & \frac{1}{(2 \pi)^{n / 2}} \int_{-\infty}^{\infty} \cdots \int_{-\infty}^{\infty} \exp \left(i t \sum x+i u g\left(x_{1}, \cdots, x_{n}\right)\right. \\
& \left.\quad-\frac{1}{2} \sum x^{2}\right) d x_{1} \cdots d x_{n} \\
= & E\left(\exp \left(i t \sum x\right)\right) \cdot \phi(t, u), \text { say, }
\end{aligned}
$$

where

$$
\begin{aligned}
\phi(t, u)= & \frac{1}{(2 \pi)^{n / 2}} \int_{-\infty}^{\infty} \cdots \int_{-\infty}^{\infty} \exp \left(\operatorname{iug}\left(x_{1}, \cdots, x_{n}\right)\right. \\
& \left.-\frac{1}{2} \sum(x-i t)^{2}\right) d x_{1} \cdots d x_{n} .
\end{aligned}
$$

Now it is given that $g\left(x_{1}, x_{2}, \cdots, x_{n}\right)$ and $g\left(x_{1}+\lambda, x_{2}+\lambda, \cdots\right.$, $\left.x_{n}+\lambda\right)$ are identically distributed and hence their corresponding characteristic functions should be identical, that is,

$$
\begin{aligned}
E\left\{\exp \left(i u g\left(x_{1}, x_{2}, \cdots, x_{n}\right)\right)\right\} & \\
= & E\left\{\exp \left(i u g\left(x_{1}+\lambda, x_{2}+\lambda, \cdots, x_{n}+\lambda\right)\right)\right\} \\
= & \frac{1}{(2 \pi)^{n / 2}} \int_{-\infty}^{\infty} \cdots \int_{-\infty}^{\infty} \exp \left(i u g\left(x_{1}+\lambda, \cdots, x_{n}+\lambda\right)\right. \\
& \left.\quad-\frac{1}{2} \sum x^{2}\right) d x_{1} \cdots d x_{n} \\
= & \frac{1}{(2 \pi)^{n / 2}} \int_{-\infty}^{\infty} \cdots \int_{-\infty}^{\infty} \exp \left(i u g\left(x_{1}, \cdots, x_{n}\right)\right. \\
& \left.\quad-\frac{1}{2} \sum(x-\lambda)^{2}\right) d x_{1} \cdots d x_{n} \\
= & \phi(-i \lambda, u) .
\end{aligned}
$$


Thus it follows from (6) that $\phi(t, u)$ which is defined only for real $t$ and real $u$ in (5) must also exist for all complex $t$, since then it represents the characteristic function of the distribution of $g\left(x_{1}, x_{2}, \cdots\right.$, $\left.x_{n}\right)$ under the assumptions of the theorem.

Again from (6) it is obvious that $\phi(t, u)$ does not involve $t$ for all complex $t$. Hence $\phi(t, u)$ being an entire function in $t$ should be free of $t$ for all real $t$ also.

Thus we have the relation

$$
E\left(\exp \left(i t \sum x+i u g\right)\right)=E\left(\exp \left(i t \sum x\right)\right) \cdot E(\exp (i u g)) .
$$

Then the stochastic independence of $x_{1}+x_{2}+\cdots+x_{n}$ and $g\left(x_{1}, x_{2}, \cdots, x_{n}\right)$ follows immediately from (7).

Proceeding exactly in the same way we can prove a corresponding theorem for the case of gamma variates which may be stated as follows:

TheOREM 2. Let $x_{1}, x_{2}, \cdots, x_{n}$ be $n$ independently and identically distributed gamma variates with the distribution function

$$
d F(x)=\frac{1}{\Gamma(p)} e^{-x} x^{p-1} d x \quad(0 \leqq x \leqq \infty) .
$$

Then the necessary and sufficient condition that the sum $\sum x$ is distributed independently of some function $g\left(x_{1}, \cdots, x_{n}\right)$ is that $g\left(x_{1}, \cdots\right.$, $\left.x_{n}\right)$ and $g\left(\lambda x_{1}, \cdots, \lambda x_{n}\right)$ should be identically distributed.

In conclusion, the author expresses his thanks to the referee for some valuable comments.

Note added in proof: It is easy to construct examples to show that for the case of independent observations from a normal (or gamma) distribution, some statistic may be distributed independently of the mean without being translation- (or scale-) invariant. A number of such examples have been given by the author in his doctoral thesis On characterizations of probability distributions and statistics, submitted to Calcutta University in 1955.

\section{REFERENCES}

1. H. Cramér, Mathematical methods of statistics, Princeton University Press, 1946, p. 94.

2. J. F. Daly, On the use of the sample range in an analogue of the student's t-test, Ann. Math. Statist. vol. 17 (1946) p. 71.

3. E. J. G. Pitman, The "closest" estimates of statistical parameters, Proc. Cambridge Philos. Soc. vol. 23 (1937) p. 212.

Indian Statistical Institute 